\title{
A point mutant of apolipoprotein A-I, V156K, exhibited potent anti-oxidant and anti-atherosclerotic activity in hypercholesterolemic C57BL/6 mice
}

\author{
Kyung-Hyun Cho ${ }^{1,6}$, Sun-Hyun Park ${ }^{1}$, \\ Jong-Min $\mathrm{Han}^{2}$, Hyoung-Chin $\mathrm{Kim}^{3}$, \\ Young-Jin Chung ${ }^{4}$, Inho Choi ${ }^{1}$ \\ and Jae-Ryong $\mathrm{Kim}^{5}$ \\ ${ }^{1}$ School of Biotechnology and Aging-associated Vascular \\ Disease Research Center \\ Yeungnam University \\ Gyeongsan 712-749, Korea \\ ${ }^{2}$ Laboratory of Lipid Metabolism \\ ${ }^{3}$ Laboratory of Experimental Animal \\ Korea Research Institute of Bioscience and Biotechnology \\ Daejeon 305-333, Korea \\ ${ }^{4}$ Department of Food and Nutrition \\ Chungnam National University \\ Daejeon 305-764, Korea \\ ${ }^{5}$ Department of Biochemistry and Molecular Biology \\ Aging-associated Vascular Disease Research Center \\ Yeungnam University \\ Daegu 705-717, Korea \\ ${ }^{6}$ Corresponding author: Tel, 82-53-810-3026; \\ Fax, 82-53-814-3026; E-mail, chok@yu.ac.kr
}

Accepted 5 February 2007

Abbreviations: HCHF, high cholesterol high fat; NBD-cholesterol, 2-(N-7-nitrobenz-2-oxa-1,3-diazol-4-yl) amino-23,24-bisnor-5-cholen$3 \beta$-ol; POPC, palmitoyloleoyl phosphatidylcholine; TBARS, thiobarbituric acid reactive substances; WT, wildtype

\begin{abstract}
In our previous study, two point mutants of apolipoprotein A-I, designated V156K and A158E, revealed peculiar characteristics in their lipid-free and lipid-bound states. In order to determine the putative therapeutic potential of these mutants, several in vitro and in vivo evaluations were conducted. In the lipid-free state, V156K showed more profound antioxidant activity against LDL oxidation than did the wildtype (WT) or A158E variants in an in vitro assay. In the lipid-bound state, V156K-rHDL showed an enhanced cholesterol delivery activity to HepG2 cells in a time-dependent manner, as compared to WT-rHDL, A158E-rHDL, and R173C-rHDL. We as-
\end{abstract}

sessed the physiological activities of the mutants in circulation, using hypercholesterolemic mice (C57BL6/J). Palmitoyloleoyl phosphatidylcholine (POPC)-rHDL preparations containing each of the apoA-I variants were injected into the mice at a dosage of $30 \mathrm{mg}$ of apoA-1/kg of body weight. Forty eight hours after injection, the sera of the V156KrHDL injected group showed the most potent antioxidant abilities in the ferric acid removal assay. The V156K-rHDL- or R173C-rHDL-injected mice showed no atherosclerotic lesions and manifested striking increases in their serum apo-E levels, as compared to the mice injected with WT-rHDL or A158E-rHDL. In conclusion, V156K-rHDL exhibited the most pronounced antioxidant activity and anti-atherosclerotic activity, both in vitro and in vivo. These results support the notion that HDL-therapy may prove beneficial due to its capacity to induce accelerated cholesterol excretion, as well as its enhanced antioxidant and anti-inflammatory effects and lesion regression effect.

Keywords: antioxidants; apolipoprotein A-I; atherosclerosis; lipoproteins, HDL; mutant proteins

\section{Introduction}

A growing body of evidence suggests that HDL may exert atheroprotective effects (Gordon et al., 1977; Linsel-Nitschke and Tall, 2005), due to its anti-oxidative and anti-inflammatory properties (Barter et al., 2004) via the augmentation of reverse cholesterol transport. The functional virtues of HDL appear to be profoundly dependent on apolipoprotein (apo) composition and on the conformation of primary apolipoproteins, including apoA-I and apoA-II (Schultz et al., 1993). Over decades, several sets of apoA-I variants, with different function and structural correlations of HDL, have been generated and characterized, and their clinical potentials have been assessed in animal and human models. This essential field is generally referred to as HDL-therapy (Newton and Krause, 2002; Brewer, 2004). Among these mutants, intravenous injections of $\mathrm{rHDL}$ containing R173C-apoA-I (apoA-I Milano $_{\text {) }}$ have been shown to have potent regression activity in a human phase II clinical trial, although the mechanism by which this 
remedy was affected has yet to be clearly elucidated (Nissen et al., 2003). A series of in vivo studies with R173C-apoA-I resulted in improvements in the pharmaceutical potential of HDL-therapy, via the use of reconstituted HDL (rHDL) with apoA-I variants (Shah et al., 1998; Chiesa et al., 2002).

Recently, we described the unique structural and functional properties of $\mathrm{V} 156 \mathrm{~K}$ and A158E (Han et al., 2005), during which we discovered that the two amino acids in helix 6 are essential to both the structure and function of rHDL. Our study also indicated that various parameters of the two mutants were markedly different in the lipid-free and lipid-bound states, thereby suggesting that the mutants might play different physiological roles in the circulation. We then prepared four kinds of rHDL with palmitoyloleoyl phosphatidylcholine (POPC) using apoA-I and its point mutants; wildtype (WT), V156K, A158E, and $\mathrm{R} 173 \mathrm{C}$, and attempted to further evaluate their in vivo therapeutic potential, as recently reported (Cho et al., 2006). WT-rHDL and R173C-rHDL were used as negative and positive controls (Nissen et al., 2003), respectively, and both of these harbored an extra 6 propeptide amino acids within their N-terminal regions, as did $\mathrm{V} 156 \mathrm{~K}$ and $\mathrm{A} 158 \mathrm{E}$. From the previous report, we determined that V156K and R173C promote anti-inflammatory function in vivo and antioxidant activities in vitro. In this report, the potent antioxidant activities of these variants were examined at lower concentrations using an in vitro system, as well as a cellular system that was able to detect the delivery of HDL-cholesterol into hepatic cells. In order to obtain further information regarding the antioxidant and anti-atherogenic potential of the V156K mutant, both in vitro and in vivo assays were conducted with hypercholesterolemic C57BL/6 mice.

\section{Materials and Methods}

\section{Recombinant apolipoproteins and synthesis of rHDL}

Wildtype proapoA-I (WT) and its mutants, V156K, $A 158 E$, and R173C, were generated via PCR based on a site-directed mutagenesis kit (Staragene, La Jolla, CA). Proteins were expressed and purified using the pET30 expression system (Novagen, Madison, WI) coupled with $\mathrm{Ni}^{2+}$-nitrilotriacetic acid column chromatography. Reconstituted HDL (rHDL) was prepared with the purified apolipoproteins (at least $95 \%$ purity) via sodium cholate dialysis at a molar ratio of 95:5:1:150, POPC:cholesterol:apoA-l: sodium cholate, as described previously by our research group (Han et al., 2005). The relevant apolipoproteins and rHDLs were characterized in a previous report (Han et al., 2005).

\section{Antioxidant assay against LDL-oxidation}

In order to determine the anti-oxidative ability of LDL, fresh human LDL $(0.05 \mathrm{ml}, 108 \mu \mathrm{g} / \mathrm{ml})$ in PBS $(\mathrm{pH}$ 7.4) was incubated for $4 \mathrm{~h}$ at $37^{\circ} \mathrm{C}$ with final $5 \mu \mathrm{M}$ of $\mathrm{CuSO}_{4}$ solution, in the presence of the indicated concentrations of apolipoproteins. Following incubation, the extent of LDL-oxidation was assessed via the measurement of the generated thiobarbituric acid reactive substances (TBARS) at $517 \mathrm{~nm}$ (Blois, 1958). In order to verify the spectroscopic data, the oxidized samples were subjected to electrophoresis on $0.5 \%$ agarose gel to compare their electromobilities (Noble, 1968). The migration of each lipoprotein (VLDL, LDL, $\mathrm{HDL}_{2}$, and $\mathrm{HDL}_{3}$ ) is known to depend on its intact charge and size. The gels were dried and the bands were stained with $0.125 \%$ Coomassie Brilliant Blue.

\section{Measurement of cholesterol delivery into HepG2 cells} Hepatic carcinoma cell (HepG2) was cultured in DMEM supplemented with $10 \%$ FBS, 2 mM glutamine, and $1 \%(\mathrm{v} / \mathrm{v})$ penicillin/streptomycin antibiotics. Cells were incubated in a chamber with an airhumidified atmosphere containing $5 \% \quad \mathrm{CO}_{2}$ at $37^{\circ} \mathrm{C}$. An aliquot of rHDL containing 22-(N-7-nitrobenz2-oxa-1,3-diazol-4-yl)amino-23,24-bisnor-5-cholen-3 $\beta$-ol (NBD-cholesterol, Molecular Probe N-1148, 70 $\mu \mathrm{g}$ of NBD-cholesterol $/ 1 \mathrm{mg}$ of apoA-I) was added to 12 -well plates $\left(4 \times 10^{5} \mathrm{cell} / \mathrm{well}\right)$ at a final concentration of $25 \mu \mathrm{M}$ in the absence of acyl-CoA:cholesterol acyltransferase (ACAT) inhibitor, in order to allow for cholesterol esterification and the detection of fluorescence. After the designated incubation time, the cell in the plate was washed with PBS and counted with a Victor $^{2}$ microplate reader (PerkinElmer) from the top reading ( $E x=488 \mathrm{~nm}, \mathrm{Em}=535$ $\mathrm{nm}$ ) without cell disruption. The parallel plate, which was incubated on a cover glass, was observed via confocal microscopy (Zeiss LSM510 Meta, Ex $=488$ $\mathrm{nm}, \mathrm{Em}=533 \mathrm{~nm}$ ) in order to compare the fluorescence intensity. The confocal visualization of NBDcholesterol uptake was conducted as previously described (Dagher et al., 2003; Kwak et al., 2005).

\section{In vivo test using hypercholesterolemic mice}

The experimental design of the in vivo test was identical to our previous report (Cho et al., 2006). In brief, normal male C57BL/6J mice (22-24 g of body weight) were obtained at 5-weeks of age from the Jackson Laboratory (Bar Habor, ME). The mice were maintained at $22 \pm 1^{\circ} \mathrm{C}$ with $55 \pm 5 \%$ humidity under a 12-h light-dark cycle. After 23 weeks of the ad libitum consumption of a CRF-1 diet (Oriental Yeast, Tokyo, Japan) containing high cholesterol and high fat $(\mathrm{HCHF}, 0.5 \%$ cholesterol $/ 15 \%$ lard $/ 0.1 \%$ of 
sodim cholate), the mice were randomly divided into 7 groups ( $n=7-12$ animals per group) and injected with $\mathrm{rHDL}$ in $0.3 \mathrm{ml}$ of PBS with $30 \mathrm{mg}$ of apoA-1/kg of body weight, as described in Table 1. After two injections given at 24-h intervals, the mice were anesthetized via enflurane inhalation, and sacrificed $48 \mathrm{~h}$ after the initial injection. During the entirety of the experimental period, the animals were cared for in accordance with the principles for the use of animals for research provided by the committee of the Korea Research Institute of Bioscience and Biotechnology (KRIBB, Daejeon, Korea).

\section{Immunodetection of serum apolipoproteins and PLTP}

Forty eight $h^{\prime}$ post-injection, blood samples were obtained from the retroorbital plexus through heparinized microhematocrit capillaries (Sigma, St. Louis, $\mathrm{MO}$ ) and collected in EDTA-treated tubes. In order to compare the expressional levels of apolipoproteins in serum, the pooled sera of each group were diluted to equal concentrations of approximately 30 -fold. The equally diluted serums were then electrophoresed via SDS-PAGE and analyzed by Western blot analysis (Eun et al., 2004) using apo-B antibody (Chemicon, \#AB742, Temecula, CA), apo-E antibody (BD Sciences \#610449, Franklin Lakes, NJ), and phospholipid transfer protein (PLTP) antibody (Abcam \#7735, Cambridge, UK). Relative band intensities were compared via band scanning using a Gel Doc ${ }^{\circledR}$ XR (Bio-Rad, Hercules, CA) with Quantity One software, version 4.5.2.

\section{Ferric reducing ability of serum assay}

In order to compare antioxidant ability of mouse serum among the groups, ferric reducing ability of serum (FRAS) was determined as previously described (Benzie and Strain, 1996). The FRAS reagents were freshly prepared via the mixing of $25 \mathrm{ml}$ of $0.2 \mathrm{M}$ acetate buffer $(\mathrm{pH} 3.6)$ with $2.5 \mathrm{ml}$ of $10 \mathrm{mM}$ 2,4,6, tripyridyl-s-triazine (TPTZ, Sigma \#T1253), and $2.5 \mathrm{ml}$ of $20 \mathrm{mM} \mathrm{FeCl}_{3} 6 \mathrm{H}_{2} \mathrm{O}$ solution. The antioxidant activities of the sera were estimated from the increase in absorbance induced by the generated ferrous ions. Freshly prepared FRAS reagent (300 $\mu \mathrm{l})$ was mixed with $10 \mu \mathrm{l}$ of mouse serum and $30 \mu \mathrm{l}$ of water. Absorbance readings were obtained at 593 $\mathrm{nm}$ every $20 \mathrm{~s}$ over a $10 \mathrm{~min}$ period.

\section{Atherosclerotic lesion analysis}

Aortic lesion formation was evaluated via a previously described method (Paigen et al., 1987) with the slight modifications described by Gulledge et al. (2003). After the mice were exsanguinated, their hearts were perfused through the left ventricle with $30 \mathrm{ml}$ of $4 \%$ paraformaldehyde and fixed for at least $24 \mathrm{~h}$. The isolated upper heart/aortic sinus was then embedded in Tissue-Tek OCT compound (Fisher Scientific, Pittsburgh, PA), and frozen. The frozen tissue blocks were placed on a cryotome model AS620 (Shandon, Pittsburgh, PA), and $10-\mu \mathrm{m}$ serial sections of the ascending aorta were collected on 3-aminopropyltriethoxysilane-coated slides until the aortic sinus appeared. The sectioned slides were stained with oil red-O and hematoxylin to visualize artherosclerotic lesions in accordance with the standard protocol (Paigen et al., 1987).

Table 1. Experimental design of rHDL injection into hypercholesterolemic mice (C57BL/6). * "

\begin{tabular}{ccccccc}
\hline group & Diet & Injected rHDL & $\mathrm{n}$ & $\begin{array}{c}\text { Body weight at } \\
\text { injection }{ }^{\dagger}(\mathrm{g})\end{array}$ & $\begin{array}{c}\text { Blood cholesterol } \\
\text { concentration before injection } \\
(\mathrm{mg} / \mathrm{dl})\end{array}$ & $\begin{array}{c}\text { Injected amount of } \\
\text { protein }^{\dagger}\end{array}$ \\
\hline $\mathrm{C}$ & Chow $^{\ddagger}$ & saline & 7 & $35.2 \pm 1.9$ & $130 \pm 28$ & Saline $0.3 \mathrm{ml}$ \\
$\mathrm{S}$ & $\mathrm{HCHF}^{\S}$ & saline & 8 & $45.3 \pm 6.4$ & $221 \pm 10$ & Saline $0.3 \mathrm{ml}$ \\
$\mathrm{WT}$ & $\mathrm{HCHF}$ & Wildtype & 8 & $41.2 \pm 4.1$ & $215 \pm 20$ & $30 \mathrm{mg} / \mathrm{kg}$ of body weight \\
V & $\mathrm{HCHF}$ & V156K & 11 & $44.6 \pm 4.3$ & $188 \pm 37$ & $30 \mathrm{mg} / \mathrm{kg}$ of body weight \\
$\mathrm{A}$ & $\mathrm{HCHF}$ & A158E & 12 & $42.8 \pm 3.7$ & $199 \pm 17$ & $30 \mathrm{mg} / \mathrm{kg}$ of body weight \\
$\mathrm{R}$ & $\mathrm{HCHF}$ & R173C & 7 & $40.2 \pm 4.5$ & $213 \pm 19$ & $30 \mathrm{mg} / \mathrm{kg}$ of body weight \\
\hline
\end{tabular}

${ }^{*}$ Data were expressed as mean \pm S.D. ${ }^{\dagger}$ The injection was done twice times 0 and $24 \mathrm{~h}$ in $0.3 \mathrm{ml}$ of PBS. ${ }^{\ddagger} \mathrm{C}$ how was CRF- 1 diet from Oriental Yeast (Tokyo, Japan). ${ }^{\S} \mathrm{HCHF}$ diet containing $0.5 \%$ cholesterol $/ 15 \%$ lard $/ 0.1 \%$ sodium cholate. "Experimental design and data were adopted from our previous report (Cho et al., 2006) 


\section{Statistical analysis}

All data were expressed as the means \pm S.D. Data was evaluated via one-way Analysis of Variance (ANOVA) using SPSS, and the differences between the means were assessed via Duncan's multiplerange test. Statistical significance was defined as $P$ $<0.05$.

\section{Miscellaneous}

LDL $(1.019<\mathrm{d}<1.063)$ was isolated via sequential ultracentrifugation (Himac CP-90 $\alpha$, Hitachi, Tokyo, Japan), at the Instrumental Analysis Center, Yeungnam University, from human serum after density adjustment via the addition of potassium bromide (Havel et al., 1955). After centrifugation, the LDL was dialyzed extensively against $10 \mathrm{mM}$ Tris- $\mathrm{HCl} / 5$

A

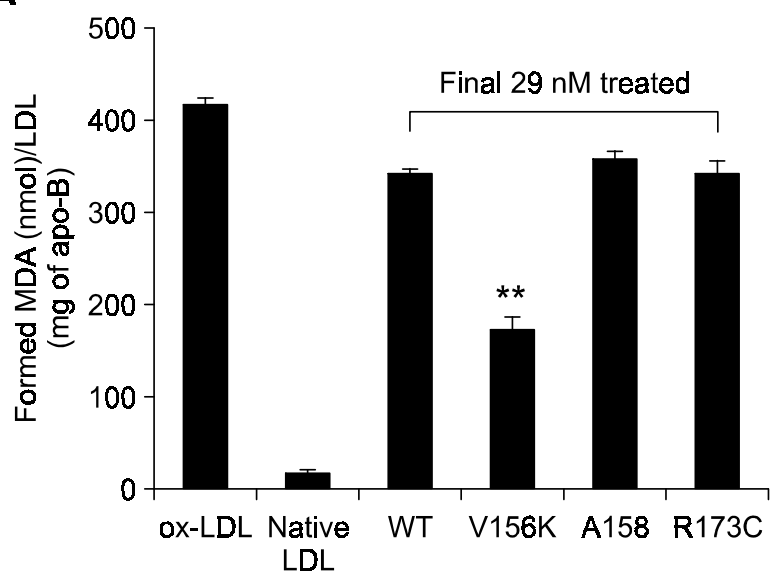

B

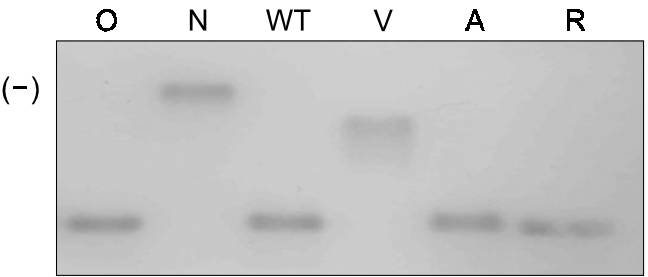

Figure 1. Anti-oxidant activity of apoA-I and its variants in lipid-free state. (A) Thiobarbituric acid reactive substance (TBARS) assay for the detection of the malondialdehyde (MDA) formed in LDL when apolipoprotein was administered at a final concentration of $29 \mathrm{nM}$ in the presence of $5 \mu \mathrm{M}$ of $\mathrm{CuSO}_{4}$. ${ }^{* *}, P<0.01$. ox-LDL, oxidized LDL (final 5 $\mu \mathrm{M} \mathrm{CuSO}{ }_{4}$ treated). (B) Comparison of the electrophoretic mobility of LDLs $(0.05 \mathrm{ml}, 108 \mu \mathrm{g} / \mathrm{ml})$, which were $29 \mathrm{nM}$ of each apolipoprotein was treated $\left(0.5 \%\right.$ agarose). Lane 0 , oxidized LDL (final $5 \mu \mathrm{M} \mathrm{CuSO}_{4}$ treated); lane $\mathrm{N}$, native $\mathrm{LDL}$ (without $\mathrm{CuSO}_{4}$ ); WT, wildtype-treated LDL; V, V156K-treated LDL; A, A158E-treated LDL; R, R173C-treated LDL.
$\mathrm{mM}$ EDTA/140 mM NaCl, at a $\mathrm{pH}$ of 7.4 , for $24 \mathrm{~h}$ in order to remove the $\mathrm{KBr}$. Protein concentration was determined using a Lowry protein assay, with the modifications described by Markwell et al. (1978) or using Bradford assay reagent (BioRad, Seoul, Korea) with BSA as a standard. The removal of endotoxin from POPC-rHDLs was verified via testing with a limulus amebocyte lysate kit (BioWhittacker cat. no. 50-647U, Walkersville, MD) in accordance with the manufacturer's suggestions.

\section{Results}

\section{Antioxidant activity against copper-mediated LDL oxidation}

HDL has been observed to exert a profound antioxidant effect against LDL oxidation, a quality which has been identified as one of HDL's primary benefits with regard to the attenuation of the progress of atherosclerotic lesion formation. In the TBARS assay, all apolipoproteins (final $290 \mathrm{nM}$ ) exhibited strong antioxidant activity with at least an $85 \%$ inhibition of malondialdehyde (MDA) generation against $\mathrm{Cu}^{2+}-$ mediated LDL oxidation, whereas probucol treatment (final $5 \mu \mathrm{M}$ ), used as a positive control, resulted in a $78 \%$ inhibition (data not shown). When $29 \mathrm{nM}$ of the protein was used for treatment, all proteins lost their activity, resulting in an inhibition rate of less than $10 \%$, with the exception of $\mathrm{V} 156 \mathrm{~K}$, which evidenced an inhibition rate of approximately $50 \%$. In order to confirm the results of TBARS, the reaction mixtures were subjected to migration on $0.5 \%$ agarose gel in order to compare electromobility. More oxidized LDL moved faster to the bottom of the gel than did the less oxidized or native LDL, as the oxidized LDL (ox-LDL) has an increased negative charge and a reduced size as the result of apo-B fragmentation (Bedwell et al., 1989). As shown in Figure 1B, when the $29 \mathrm{nM}$ of apoA-I treated LDL was applied, all LDL moved to the bottom, to the same position as was observed with the oxidized LDL, with the exception of the V156K-treated LDL. This indicates that all proteins lost their activity at lower concentrations. The V156K-treated LDL proved recalcitrant to move down, thus suggesting that V156K evidenced potent antioxidant activity under identical concentrations. This result was very consistent with the results of the TBARS assay (Figure $1 \mathrm{~A})$.

\section{V156K-rHDL showed the strongest cholesterol delivery activity}

In order to facilitate the excretion of cholesterol from the circulation, the major beneficial role of $\mathrm{HDL}$ 
A

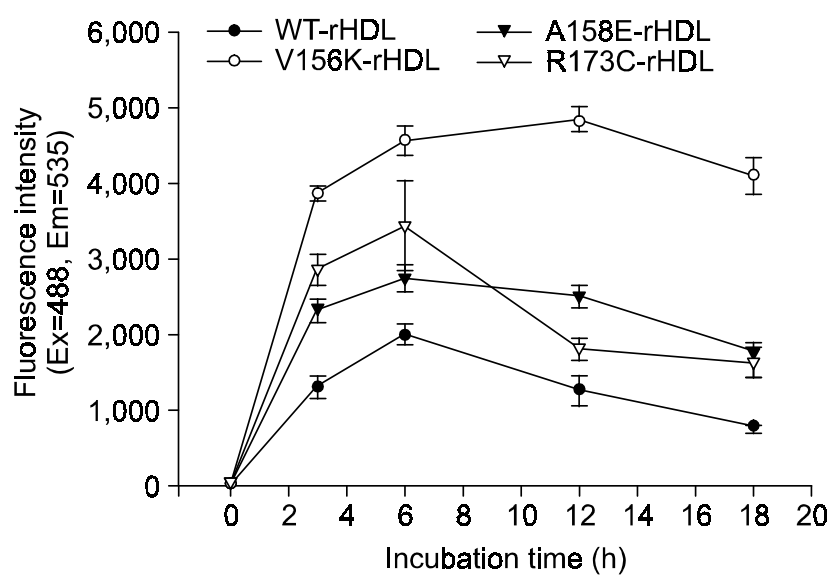

B
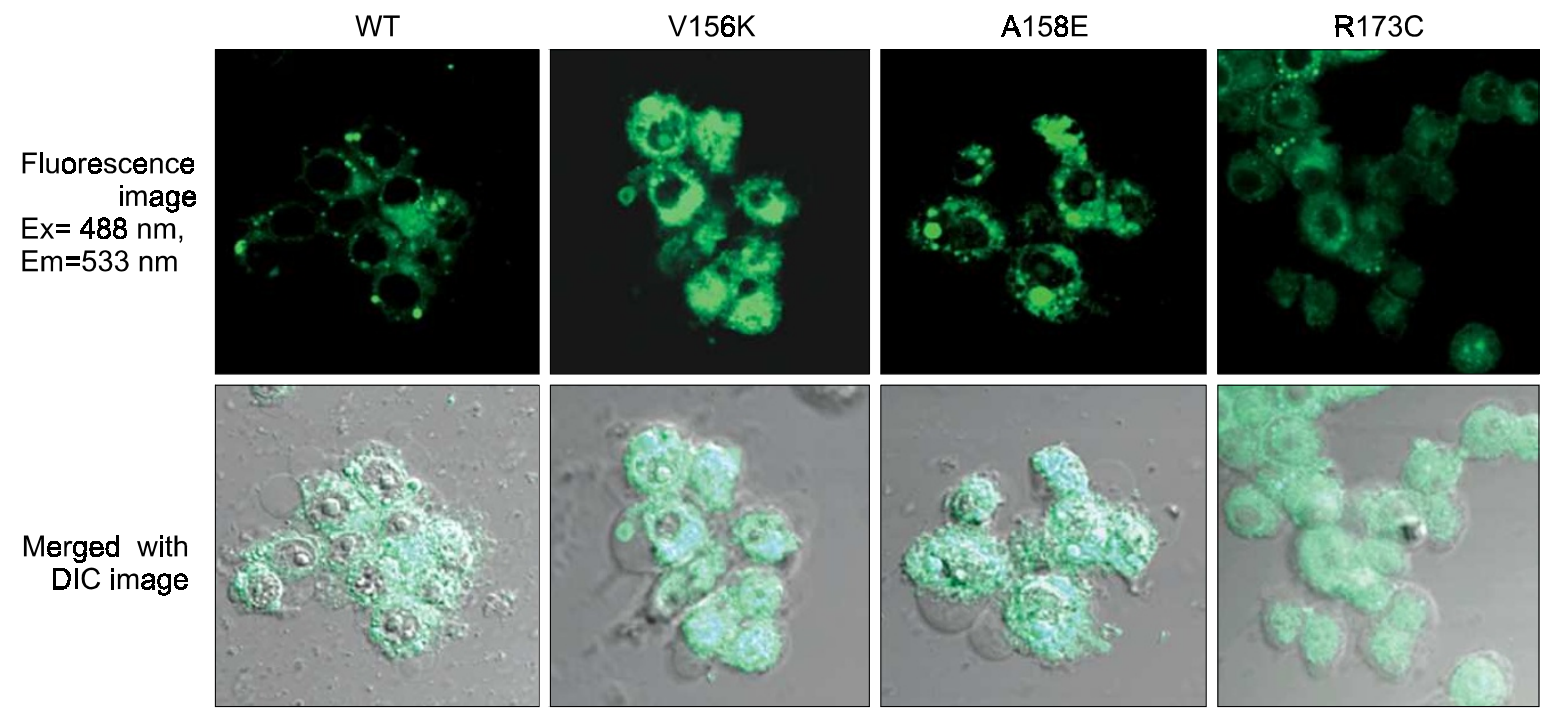

Figure 2. Measurement of cholesterol delivery activity into HepG2 cell. After the administration of $\mathrm{rHDL}$ containing NBD-cholesterol $(70 \mu \mathrm{g}$ of NBD-cholesterol per $1 \mathrm{mg}$ of apoA-I) to cultured cells and incubation at $37^{\circ} \mathrm{C}$ in an atmosphere containing $5 \% \mathrm{CO}_{2}$. (A) Detected fluorescence signal at designated time after the removal of medium and cell rinsing by optical microplate reader ( $E x=488 \mathrm{~nm}, E \mathrm{~m}=535 \mathrm{~nm}$ ). Data were averaged from three independent assays. (B) Confocal scanning microscopic images of the rHDL-treated HepG2 cells (Ex $=488 \mathrm{~nm}, \mathrm{Em}=533 \mathrm{~nm}$ ). During the 18 $h$ incubation period, the NBD-cholesterol was delivered to the inside of the cells and esterified in cytosol for detection.

involves the delivery of cholesterol into hepatic tissue from the peripheral cells via reverse cholesterol transport (Glomset, 1968). In order to evaluate this cholesterol delivery activity, NBD-cholesterol was used as a fluorescent marker for the cholesterol analogue located in the core of the rHDL. After the treatment of the fluorescence-labeled rHDL to HepG2 cells, V156K-rHDL evidenced superior delivery efficiency to other mutant-rHDL variants, in a time-dependent manner. During the incubation period, V156K-rHDL evidenced almost two-fold higher delivery efficiency than was seen with A158E-rHDL, and almost three-fold higher delivery efficiency as compared to the WT-rHDL, as shown in Figure 2A. R173C-rHDL evidenced more potent activity than A158E-rHDL until $6 \mathrm{~h}$ of incubation time. In the early stages of incubation, at approximately $6 \mathrm{~h}$, all rHDLs evidenced maximum cholesterol delivery activity, with the exception of V156K-rHDL, which achieved maximum activity at $12 \mathrm{~h}$ of incubation. Fluorescent microscopic examination supported the observation that the V156K-rHDL-treated cells exhibited the strongest fluorescence signal, and these signals were also detected in the cytosol, as shown in Figure 2B. 
The R173C-rHDL-treated cells evidenced much weaker intensity, closer to that of WT- rHDL or A158E$\mathrm{rHDL}$, because the microscopic observation was conducted at $18 \mathrm{~h}$.

\section{Change of apolipoprotein expressional levels}

In order to compare the changes in the levels of apolipoprotein expression as the result of the injection of each rHDL, mice serum apolipoproteins were analyzed via Western blot. Although this difference was not significant between groups, each rHDL-injected mouse group evidenced slightly increased mouse apoA-I expressional levels as compared to salineinjected mice (group $S$, data not shown). The mice consuming the HCHF-diet evidenced apo-B 48 level two-fold that of the normal chow-fed group (top panel of Figure 3), thereby indicating that serum LDL levels had been increased by up to two-fold during the long-term HCHF-diet consumption period. Interestingly, the groups of mice injected with WT-rHDL (lane WT) or A158E-rHDL (lane A) evidenced significantly increased apo-B levels, of up to 2.6 and 3.2-fold that seen in the saline-injected group (lane S), whereas the V156K-rHDL (lane V) or R173CrHDL (lane R) injected groups evidenced no significant increases in apo-B levels. Immunodetection

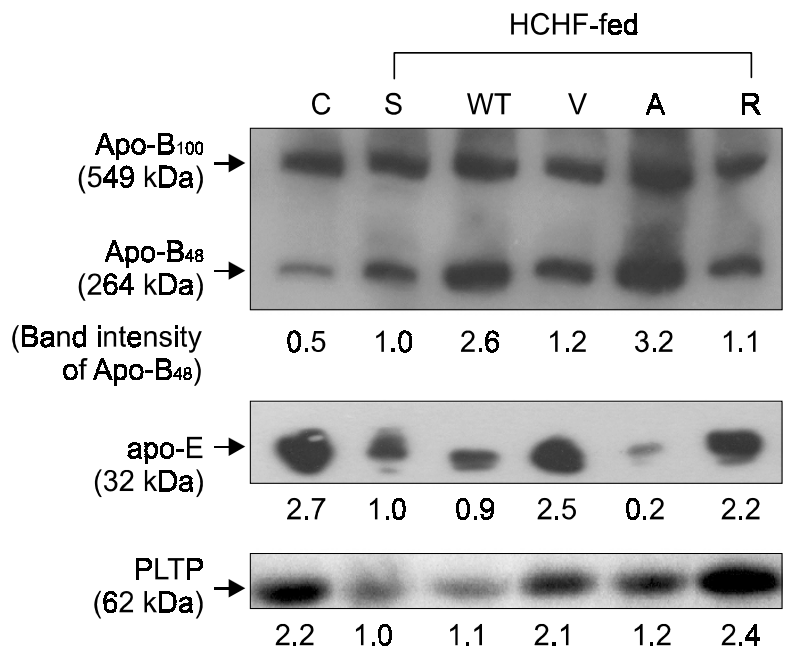

Figure 3. Immunodetection of apolipoproteins in mice serum. Western blot analysis with mouse apo-B antibody (264 kDa for $\left.a p o B_{48}\right)$, apo-E (32 kDa) antibody, and PLTP (62 kDa) antibody. The lower numbers indicate the relative band intensity in comparison with lane $S$ (saline-injected group). In V156K-rHDL and R173C-rHDL injected mice, the expressional levels of serum apo-E and PLTP were increased significantly (lanes $\mathrm{V}$ and $\mathrm{R}$ ). Lane $\mathrm{C}$, saline-injected mice normal chowfed mice; lane S, saline-injected HCHF-fed mice; lane WT, WT-rHDL injected HCHF-fed mice; lane V, V156K-rHDL injected HCHF-fed mice; lane A, A158E-rHDL injected HCHF-fed mice; lane R, R173C-rHDL injected HCHF-fed mice. using a mouse apo-E specific antibody showed that HCHF-diet consumption (group S) induced a marked reduction of apo-E (32 $\mathrm{kDa})$ levels in serum at 48 h' post-injection, as was shown in the middle panel of Figure 3 (lane S). V156K-rHDL or R173C-rHDL injection, however, caused a remarkable increase of apo-E, by up to 2.5-fold (lane V), whereas the WTrHDL or A158E-rHDL injected groups evidenced minimized apo-E expressional levels. HCHF-fed mice (group S) manifested notably reduced PLTP bands as compared to the normal chow-fed group, which suggests that PLTP may exert anti-atherogenic effects in murine models. The intensity of the PLTP band increased distinctly, reaching levels of approximately 2.1-2.4 fold that in the V156K-rHDL injected (lane V) and R173C-rHDL injected (lane R) mice, as compared to the levels measured in the WT-rHDL or A158E-rHDL-injected groups.

\section{V156K-rHDL infused serum evidenced the strongest antioxidant ability}

The FRAS assay is known to be a rapid and reproducible method for determining the molar concentration of the antioxidant present (Benzie and Strain, 1996). We conducted the FRAS assay with mice sera in order to compare antioxidant effects between the groups. The V156K-rHDL-infused serum evidenced the most profound antioxidant activity in terms of serum ferric removal ability, as shown in Figure 4. Up to $10 \mathrm{~min}$ of incubation, V156K-injected serum evidenced dramatic increases in absorbance at 593

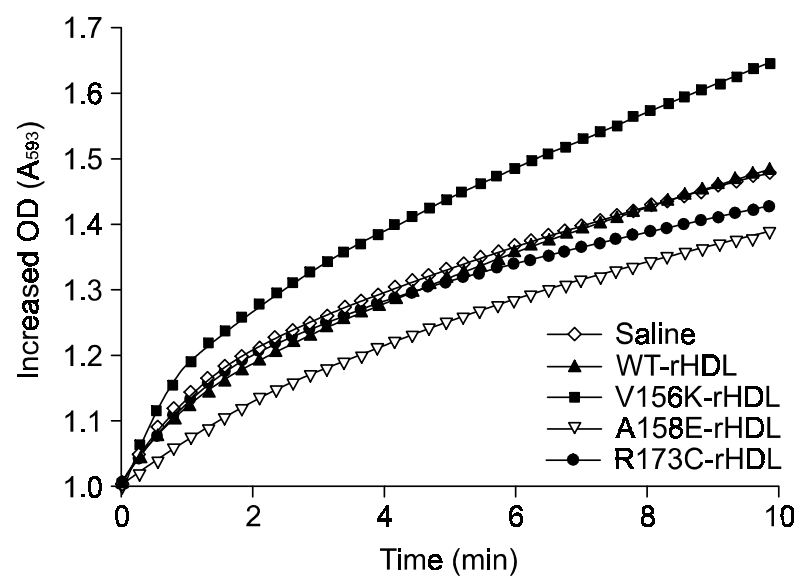

Figure 4. Comparison of antioxidant ability from ferric reducing ability of serum (FRAS). Up to $10 \mathrm{~min}$ of incubation, V156K-injected serum evidenced dramatic increases in absorbance at $593 \mathrm{~nm}\left(\mathrm{~A}_{593}\right)$ of up to $64 \%$ more than the initial values. However, the WT-rHDL, R173C$\mathrm{rHDL}$, and A158E-rHDL injected mice evidenced increases of $48 \%$, $42 \%$, and $38 \%$, respectively, more than each of their initial values. The data were expressed as the means $\pm S D$ from three independent measurements with duplicate samples. 
$\mathrm{nm}\left(\mathrm{A}_{593}\right)$ of up to $64 \%$ more than the initial values. However, the WT-rHDL, R173C-rHDL, and A158E$\mathrm{rHDL}$ injected mice evidenced increases of $48 \%$, $42 \%$, and $38 \%$, respectively, more than each of their initial values (Figure 4), thus indicating that those mice evidenced weaker anti-oxidative ability than did V156K-rHDL in the blood. This result is quite consistent with the results of the in vitro antioxidant assay (Figure 1), in which V156K was determined to evidence the most potent antioxidant activity against copper-mediated oxidation.

\section{Atherosclerotic lesions disappeared in the V156K-rHDL and R173C-rHDL mice}

After 23 weeks of HCHF-feeding, relatively thick lesions were detected in the aortic sinus sections from the saline-injected mice, as evidenced by the oil-red $O$ staining images shown in Figure 5. Although atherosclerotic lesions were not observed throughout the entirety of the sinus region, the oil red O-stained region was thickened and had obviously accumulated locally in the aortic sinus, as was also observed in the saline-injected group. The V156K-rHDL-injected group evidenced no such accumulated lesions, such that the lesions had almost completely disappeared. This result was similar to what had been observed with the R173C-rHDL injected group. These results could be compared with those of the WT-rHDL or A158E-rHDL-injected groups, which evidenced much weaker inhibition activity. Although the statistical significance of lesion size distribution was not determined in the current study, the obtained data correlate well with the expressional levels of serum apo-E data acquired via immunodetection (Figure 3). Apo-E is abundantly expressed by macrophages in both human and rabbit atherosclerotic plaques (Rosenfeld et al., 1993). Our results indicate that immobilized apo-E located inside the cores of foam cells might be secreted into the blood stream simultaneously with lesion removal in the V156KrHDL or R173C-rHDL-injected groups.

\section{Discussion}

HDL-cholesterol levels are known to be associated inversely with reductions of cardiovascular risk, and this has been corroborated by the results of a number of epidemiologic studies (Assmann and
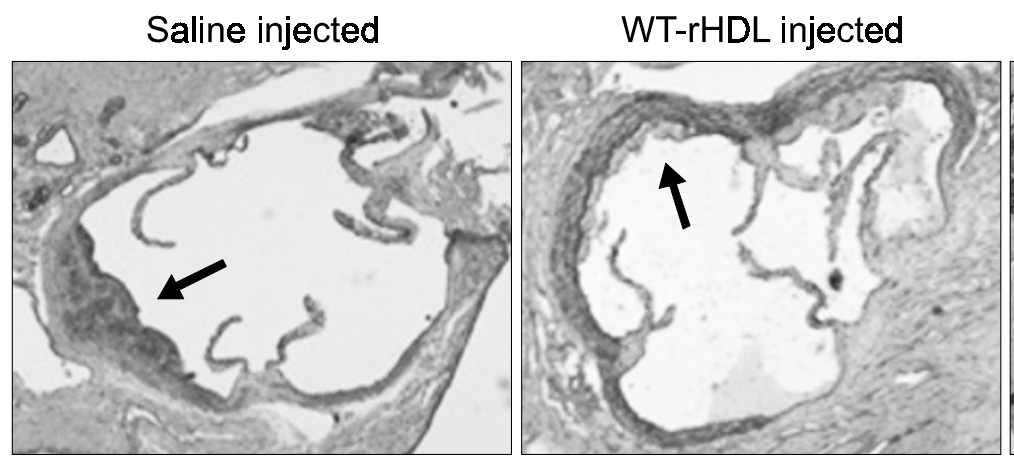

\section{V156K-rHDL injected}

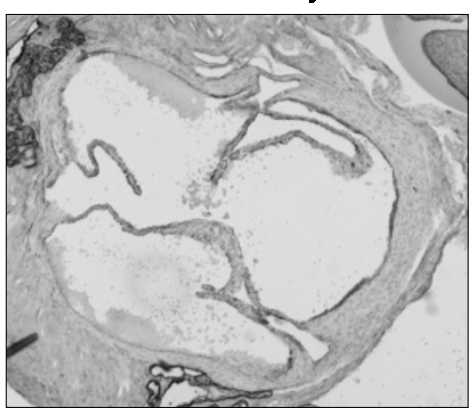

A158E-rHDL injected

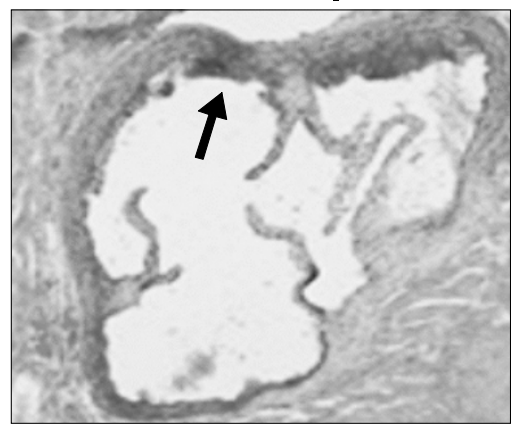

R173C-rHDL injected

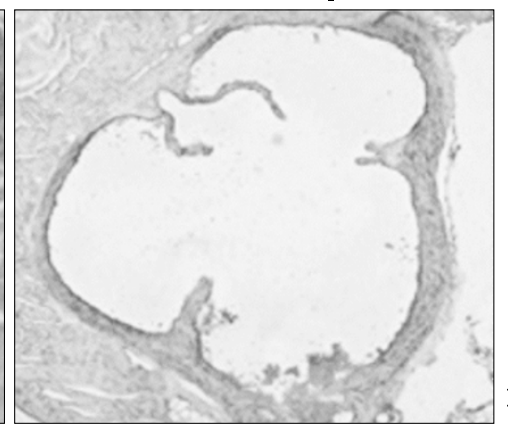

$\times 40$ magnifications

Figure 5. Representative photomicrograph of cross-sectioned mouse aortic sinus and visualization of atherosclerotic lesions. Representative sections were stained with oil red $\mathrm{O}$ and counterstained with hematoxylin, allowing for the visualization of the lipid area, which was observed as a red region ( $\times 40$ magnification). After 23 weeks of consumption of an HCHF-diet, a locally thickened fatty streak lesion was detected in the saline-injected group. The lesion was almost undetectable in the V156K-rHDL and R173C-rHDL injected groups, as compared with the other rHDL-injected groups. 
Schulte, 1992), although the exact mechanism underlying HDL's anti-atherogenic potential remains uncertain. One of the widely-accepted explanations is that inhibitory activity against LDL oxidation is a firmly established virtue of $\mathrm{HDL}$, representing a potent anti-atherogenic factor. This antioxidant activity is known to impart a variety of beneficial cellular effects, i.e., prevention of oxidized-LDL uptake by macrophages, the inhibition of endothelial cell adhesion molecule expression, and the promotion of cholesterol efflux from macrophages in atherosclerotic lesions. In this report, the two antioxidant assays in vitro (Figrue 1) and in vivo (Figure 4) confirmed that $\mathrm{V} 156 \mathrm{~K}$ had the most profound antioxidant activity among the wildtype and mutants, both in the lipid-free (Figure 1) and rHDL states (Figure 4). The antioxidant activity of the apoA-I variants, apoA-I $I_{\text {Milano }}$ and apoA-I $I_{\text {Paris, }}$ in the lipidbound state has been fairly well established (Bielicki and Oda, 2002), but the same activity of apoA-I in the lipid-free state has yet to be definitively elucidated. Our current results show that the antioxidant activity of HDL derives, at least in part, from apoA-I itself without phospholipid interaction. However, Pownall and Ehnholm (2005) emphasized that phosphatidylcholine is an active agent in HDL-therapy, rather than apoA-I or its variants. Our current data, however, suggest that POPC evidences different therapeutic activity depending on its apoA-I variant as a HDL constituent. Thus our results show that apoA-I has potent antioxidant activity in its lipid-free state, as shown in Figure 1, and this represents one of the more notable beneficial functions of HDL.

The hepatic uptake of plasma HDL-derived cholesterol and cholesteryl ester into the liver for excretion into the bile (Acton et al., 1996) has been identified as an essential pathway for the maintenance of cholesterol homeostasis. The delivery measurement (Figure 2A) indicates that the delivery activity of V156K-rHDL lasted longer than was seen with the other HDL variants, thereby suggesting that V156K has better protein stability in cell culture media and superior interaction ability with a putative HDL-receptor, such as scavenger receptor B-I (Acton et al., 1996), of the HepG2 cell.

It has been reported that apo-E is expressed abundantly by macrophages in human and rabbit atherosclerotic plaques (Rosenfeld et al., 1993) and they are co-localized inside of fatty streak lesions, especially the necrotic core, because the macrophage is the principal source of apo-E in the lesion. As shown in the middle panel of Figure 3, the V156K-rHDL and R173C-rHDL-injected groups evidenced remarkable increases in apo-E levels in the circulating blood, whereas the A158E-rHDL-injected group evidenced a band intensity 12-fold weaker (lane A of Figure 3). In comparison with the observed changes in the levels of apo-A-I, this dramatic increase in serum apo-E levels allows for speculation that the immobilized apo-E located within the core of the foam cell may be released into the bloodstream simultaneously with lesion regression.

Another interesting point is the increase of serum PLTP in the V156K-rHDL or R173C-rHDL- injected group, which was almost two-fold of the WT-rHDL or A158E-rHDL-injected groups, as shown in the bottom panel of Figure 3. Serum PLTP performs crucial functions in HDL remodeling and the maintenance of HDL levels via the transfer of phospholipids from remnant lipoproteins. The relationship between PLTP and CHD remains a matter of some controversy, i.e., high PLTP activity has been identified as a risk factor for CHD (Schlitt et al., 2003) but PLTP mass also appears to be a protective factor against CHD (Yatsuya et al., 2004). A study of 1,102 CHD patients indicated that the concentration of serum PLTP was significantly inversely related with the risk of cardiovascular disease (Yatsuya et al., 2004). This finding shows that immuno-detected PLTP masses increased in cases of V156K-rHDL or R173C-rHDL injection, thereby suggesting that increases in PLTP mass might be correlated with anti-atherosclerotic processes. These results can be interpreted such that V156K-rHDL, like R173C-rHDL may constitute a good candidate for HDL-therapy, whereas WT-rHDL and A158ErHDL may not be effective HDL-therapy agents.

With regard to the regression effect of V156K-rHDL and R173C-rHDL, the regression effects appeared relatively rapid despite the low dosage of protein injection as compared to the previous reports of Shah et al. $(1998,2001)$. The remarkable regression effects were observed from 18 injections of $40 \mathrm{mg} / \mathrm{kg}$

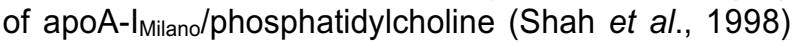
and a single injection of $400 \mathrm{mg} / \mathrm{kg}$ of apoA-I Milano/ dipalmitoyl phosphatidylcholine (DPPC) (Shah et al., 2001). However, it is hard to directly compare the current results with the results of the Shah group, as there are several different aspects inherent to the experimental design, including the model mouse (normal C57BL/6 vs apo-E deficient mice), the type of phospholipid in rHDL (POPC vs DPPC), protein dosage (30 mg/kg vs $400 \mathrm{mg} / \mathrm{kg}$ of body weight), and injection times. Normal C57BL/6 mice were relatively resistant to the progression of fatty streak lesion formation, as compared to the apo-E deficient mice; they evidenced relatively smaller and locally thickened lesions despite the consumption of the $0.5 \%$ cholesterol-diet for 23 weeks, as shown in Figure 5. However, apo-E deficient mice showed very thick lesion after only 9 weeks of consumption of regular mouse chow (Zhang, 1992). 
Similarly, in addition, Kaul et al. (2003) reported that the intramural delivery of ETC-216, apoA-I Milano and phospholipid complex, with low dosage $(0.22$ $\mathrm{mg} / \mathrm{kg}$ of body weight) into domestic swine resulted in a striking regression effect. Although the delivery method and injection times are not the same as are listed in our current report, this result demonstrated a profound regression effect in non-transgenic animal receiving only a single administration. These results allow speculating that normal animals may have a different mechanism of atherosclerotic lesion formation and removal as the result of HDL-therapy than is exploited in apo-E- deficient mice.

Our current findings can be summarized as follows: potent antioxidant activity, cholesterol-excretion ability, and fatty-streak lesion regression effects were detected for V156K-apoA-I in both the lipid-free and lipid-bound states in cellular and animal models. From the standpoint of therapeutic potential, several coincidental points were found between $\mathrm{V} 156 \mathrm{~K}$ and R173C as compared to WT and A158E. V156K and $\mathrm{R} 173 \mathrm{C}$ in the lipid free-state evidenced a superior antioxidant activity against copper-mediated LDL oxidation and cholesterol delivery activity into HepG2 cells as compared to WT and A158E. The injection of V156K-rHDL or R173C-rHDL induced enhancements of anti-oxidant activity and atherosclerotic lesion removal effects with increases in serum apo-E and PLTP.

In conclusion, our results with blood infusions of V156K-rHDL supported the benefit of HDL-therapy hypothesis (Newton and Krause, 2002), which posits that rHDL exerts an anti-atherosclerotic effect via the acceleration of reverse cholesterol transport, in addition to its anti-oxidant, anti-inflammatory, and antiatherosclerotic effects.

\section{Acknowledgement}

This work was supported in part by grant RO1- 2002000-00176-0 from the Basic Research Program of the Korea Science and Engineering Foundation (KOSEF), and was supported by the KOSEF through the Aging-associated Vascular Disease Research Center at Yeungnam University (R13-2005-005-01003-0 (2006)).

\section{References}

Acton S, Rigotti A, Landsculz KT, Xu S, Hobbs HH, Krieger $\mathrm{M}$. Identification of scavenger receptor SR-BI as a high density lipoprotein receptor. Science 1996;271:518-20

Assmann G, Schulte H. Relation of high-density lipoprotein cholesterol and triglycerides to incidence of atherosclerotic coronary artery disease (the PROCAM experience). Prospective cardiovascular munster study. Am J Cardiol 1992; 70:733-7
Barter PJ, Nicholls S, Rye KA, Anantharamaiah GM, Navab M, Fogelman AM. Antiinflammatory properties of HDL. Circ Res 2004;95:764-72

Bedwell S, Dean RT, Jessup W. The action of defined oxygen-centred free radicals on human low-density lipoprotein. Biochem J 1989;262:707-12

Benzie IF, Strain JJ. The ferric reducing ability of plasma (FRAP) as a measure of "antioxidant power": the FRAP assay. Anal Biochem 1996;239:70-6

Bielicki JK, Oda MN. Apolipoprotein A-IMilano and apolipoprotein A-IParis exhibit an antioxidant activity distinct from that of wild-type apolipoprotein A-I. Biochemistry 2002;41: 2089-96

Blois MS. Antioxidant determinations by the use of a stable free radical. Nature 1958;181:1199-200

Brewer HB. High-density lipoproteins: a new potential therapeutic target for the prevention of cardiovascular disease. Arterioscler Thromb Vasc Biol 2004;24:387-91

Chiesa G, Monteggia E, Marchesi M, Lorenzon P, Laucello M, Lorusso V, Di Mario C, Karvouni E, Newton RS, Bisgaier $\mathrm{CL}$, Franceschini G, Sirtori CR. Recombinant apolipoprotein A-I $\mathrm{I}_{\text {(Milano) }}$ infusion into rabbit carotid artery rapidly removes lipid from fatty streaks. Circ Res 2002;90:974-80

Cho KH, Park SH, Han JM, Kim HC, Choi YK, Choi I. ApoA-I mutants V156K and R173C promote anti-inflammatory function and antioxidant activities. Eur J Clin Invest 2006;36: 875-82

Dagher G, Donne N, Klein C, Ferre P, Dugail I. HDL-mediated cholesterol uptake and targeting to lipid droplets in adipocytes. J Lipid Res 2003;44:1811-20

Eun JP, Choi HY, Kwak YG. Proteomic analysis of human cerebral cortex in epileptic patients. Exp Mol Med 2004;36: $185-91$

Glomset JA. The plasma lecithin:cholesterol acyltransferase reaction. J Lipid Res 1968;9:155-67

Gordon T, Castelli WP, Hjortland MC, Kannel WB, Dawber TR. High density lipoprotein as a protective factor against coronary heart disease. Am J Med 1977;62:707-14

Gulledge AA, Mcshea C, Schwartz T, Koch G, Lord ST. Effects of hyperfibrinogenemia on vasculature of C57BL/6 mice with and without atherogenic diet. Arterioscler Thromb Vasc Biol 2003;23:130-5

Han JM, Jeong TS, Lee WS, Choi I, Cho KH. Structural and functional properties of $\mathrm{V} 156 \mathrm{~K}$ and $\mathrm{A} 158 \mathrm{E}$ mutants of apolipoprotein A-I in the lipid-free and lipid-bound states. J Lipid Res 2005;46:589-96

Hansson GK. Inflammation, atherosclerosis, and coronary artery disease. N Engl J Med 2005;352:1685-95

Havel RJ, Eder HA, Bragdon JH. The distribution and chemical composition of ultracentrifugally separated lipoproteins in human serum. J Clin Invest 1955;34:1345-53

Kaul S, Rukshin V, Santos R, Azarbal B, Bisgaier CL, Johansson J, Tsang VT, Chyu KY, Cercek B, Mirocha J, Shah PK. Intramural delivery of recombinant apolipoprotein A- 
IMilano/phospholipid complex (ETC-216) inhibits in-stent stenosis in porcine coronary arteries. Circulation 2003; 107:2551-4

Kwak JO, Kim HW, Oh KJ, Kim DS, Han KO, Cha SH. Co-localization and interaction of organic anion transporter 1 with caveolin-2 in rat kidney. Exp Mol Med 2005;37:204-12

Linsel-Nitschke P, Tall AR. HDL as a target in the treatment of atherosclerotic cardiovascular disease. Nat Rev Drug Discov 2005;4:193-205

Markwell MA, Haas SM, Bieber LL, Tolbert NE. A modification of the Lowry procedure to simplify protein determination in membrane and lipoprotein samples. Anal Biochem 1978; $87: 206-10$

Newton RS, Krause BR. HDL therapy for the acute treatment of atherosclerosis. Atherosclerosis Suppl 2002;3:31-8

Nissen SE, Tsunoda T, Tuzcu EM, Schoenhagen P,Cooper CJ, Yasin M, Eaton GM, Lauer MA, Sheldon WS, Grines CL, Halpern S, Crowe T, Blankenship JC, Kerensky R. Effect of recombinant apoA-I Milano on coronary atherosclerosis in patients with acute coronary syndromes. J Am Med Assoc 2003;290:2292-300

Noble RP. Electrophoretic separation of plasma lipoproteins in agarose gel. J Lipid Res 1968;9:693-700

Paigen B, Morrow A, Holmes PA, Mitchell D, Williams RA. Quantitative assessment of atherosclerotic lesions in mice. Atherosclerosis 1987;68:231-40

Pownall HJ, Ehnholm C. Enhancing reverse cholesterol transport: the case for phosphatidylcholine therapy. Curr Opin Lipidol 2005;16:265-8
Rosenfeld ME, Butler S, Ord VA, Lipton BA, Dyer CA, Curtiss LK, Palinski W, Witztum JL. Abundant expression of apoprotein $\mathrm{E}$ by macrophages in human and rabbit atherosclerotic lesions. Arterioscler Thromb 1993;13:1382-9

Schlitt A, Bickel C, Thumma P, Blankenberg S, Rupprecht $\mathrm{HJ}$, Meyer J, Jiang XC. High plasma phospholipid transfer protein levels as a risk factor for coronary artery disease. Arterioscler Thromb Vasc Biol 2003;23:1857-62

Schultz JR, Verstuyft JG, Gong EL, Nichols AV, Rubin EM. Protein composition determines the anti-atherogenic properties of HDL in transgenic mice. Nature 1993;365:762-4

Shah PK, Nilsson J, Kaul S, Fishbein MC, Ageland H, Hamsten A, Johansson J, Karpe F, Cercek B. Effects of recombinant apolipoprotein A-I Milano on aortic atherosclerosis in apolipoprotein E-deficient mice. Circulation 1998;97:780-5

Shah PK, Yano J, Reyes O, Chyu KY, Kaul S, Bisgaier CL, Drake S, Cercek B. High-dose recombinant apolipoprotein

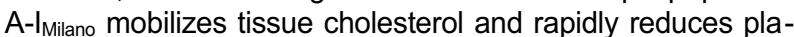
que lipid and macrophage content in apolipoprotein E-deficient mice. Circulation 2001;103:3047-50

Yatsuya H, Tamakoshi K, Hattori H, Otsuka R, Wada K, Zhang $\mathrm{H}$, Mabuchi T, Ishikawa M, Murata C, Yoshida T, Kondo T, Toyoshima $\mathrm{H}$. Serum phospholipid transfer protein mass as a possible protective factor for coronary heart diseases. Circ J 2004;68:11-6

Zhang SH, Reddick RL, Piedrahita JA, Maeda N. Spontaneous hypercholesterolemia and arterial lesions in mice lacking apolipoprotein E. Science 1992;258:468-47 